EESTI NSV TEADUSTE AKADEEMIA TOIMETISED, 26. KÖIDE

FOOSIKA O MATEMAATIKA. 1977, NR. 3

ИЗВЕСТИЯ АКАДЕМИИ НАУК ЭСТОНСКОИ ССР. ТОМ 26 ФИЗИКА * МАТЕМАТИКА, 1977, № 3

\title{
ОБ АНАЛОГИИ МЕЖДУ НЕРЕЛЯТИВИСТСКОЙ ТЕОРИЕЙ ГРАВИТАЦИИ И ТЕОРИЕЙ ЭЛЕКТРОМАГНЕТИЗМА
}

A. KOPPEL. MITTERELATIVISTLIKU GRAVITATSIOONITEOORIA JA ELEKTROMAGNETVALJA TEOORIA ANALOOGIAST

A. KOPPEL. ON THE ANALOGY BETWEEN THE NON-RELATIVISTIC GRAVITATIONAL THEORY AND THEORY OF ELECTROMAGNETIC FIELD

В данной работе обсудим некоторые общие свойства уравнений нерелятивистского (НР) гравитационного поля (Г-поля), вытекающих из подхода Кереса $\left[{ }^{1-3}\right]$, запишем эти уравнения в виде, формально одинаковом с уравнениями Максвелла-Лоренца в квазистационарном случае электромагнитного поля (ЭМ-поля), введем в случае НР Г-поля ряд величин, аналогичных электромагнитным величинам, и рассмотрим применение лагранжева и гамильтонова формализмов в обсуждаемой обобщенной теории НР Г-поля.

1. Согласно Х. Кересу $\left[{ }^{1-3}\right]$, свойства НР Г-полей выражаются в свойствах искривленных 4-пространств $\widetilde{\nabla}_{4}$ с аффинной связностью, но с вырожденной метрикой $(0,+,+,+)$. Для 4-пространств $\widetilde{\nabla}_{4}$ сингулярные метрические формы типа *

$$
\mathrm{d} s^{2}=\widetilde{v}_{s} \widetilde{v}^{s} \mathrm{~d} t^{2}+2 \widetilde{v}_{s} \mathrm{~d} \widetilde{x}^{s} \mathrm{~d} t+\tilde{\gamma}_{s p} \mathrm{~d} \tilde{x}^{s} \mathrm{~d} \tilde{x}^{p} \equiv \widetilde{g}_{\mu v} \mathrm{~d} \tilde{x}^{\mu} \mathrm{d} \tilde{x}^{v}
$$

получаются предельным переходом $c \rightarrow \infty$ из соответствующих релятивистских метрических форм для римановых $V_{4}$, описывающих релятивистские Г-поля в определенных системах отсчета с ньютоновым временем (в т. н. $G$-системах). Общие уравнения НР Г-поля также получаются как предельный вид релятивистских уравнений Г-поля, записанных в $G$-системах.

Имеем [ $\left.{ }^{4}\right]$ формулу

$$
\widetilde{R}_{\mu v}=(1 / 2) \tilde{\lambda}_{\mu v}
$$

где $\widetilde{R}_{\mu v} \equiv \lim _{c \rightarrow \infty} R_{\mu v}$ толкуются как компоненты тензора Риччи для $\widetilde{\nabla}_{4}$, $\tilde{\lambda}_{\mu v} \equiv \lim _{c \rightarrow \infty}\left[\frac{16 \pi G}{c^{4}}\left(T_{\mu \nu}-\frac{1}{2} g_{\mu v} T\right)\right], T_{\mu v}$ - составляющие тензора энергииимпульса для источников Г-поля. Конкретный вид уравнений (2) существенно зависит от структуры релятивистского тензора энергии-импульса материи. В случае т. н. обычной материи ( $T_{\mu \nu}$ описывает идеальную жидкость или ЭМ-поле) только компонента $T_{00}$ дает ненулевой

* В данной работе преческие индексы пробегают значения $0,1,2$, 3, а латинские - значения $1,2,3$. По индексам в квадратных скобках проводится альтернирование. Запятая с индексом означает дифференцирование по соответствующей координате. 
вклад в $\tilde{\lambda}_{\mu v}$, и $\tilde{\nabla}_{3}$ с метрическим тензором $\tilde{\gamma}_{s p}$ является евклидовым. После введения декартовых координат в $\widetilde{\nabla}_{3}$ уравнения (2) принимают вид

$$
\begin{gathered}
\tilde{v}_{s, s, 0}-(1 / 2)\left(\tilde{v}_{p} \tilde{v}_{p}\right)_{, s, s}+\widetilde{v}_{[p, s]} \tilde{v}_{[p, s]}=4 \pi \tilde{G}, \\
\tilde{v}_{[i, s], s}=0,
\end{gathered}
$$

где $\tilde{\mathrm{e}}$ - плотность вещества. Подчеркнем, что без ad hoc дополнительных условий далее ограничивать вид этих уравнений нельзя. Поэтому ниже и рассмотрим содержание уравнений (3)-(4) в общем случае.

Движение свободной пробной частицы в НР Г-поле определяется геодезическими линиями в $\widetilde{\nabla}_{4}$. В силу этото ускорение пробной частицы относительно декартовой координатной системы удовлетворяет уравнениям

$$
\frac{\mathrm{d} \tilde{u}_{k}}{\mathrm{~d} t}=-\tilde{v}_{k, 0}+\frac{1}{2}\left(\tilde{v}_{p} \tilde{v}_{p}\right)_{, k}+2 \tilde{u}_{s} \tilde{v}_{[s, k]} .
$$

Если хотя бы для одной тройки индексов

$$
\tilde{v}_{[i, j], k} \neq 0,
$$

то в этом случае НР Г-поле является неньютоновым (вихревым). Только при $\widetilde{v}_{[i, j], k}=0$ получается ньютонова теория гравитации $\left[{ }^{2,4}\right]$.

2. Запишем систему уравнений, формально совпадающих при надлежащем выборе единиц с уравнениями Максвелла-Лоренца при $\frac{\partial}{\partial t} \boldsymbol{e}=0$, т. е. без учета тока смещения $\left[{ }^{5-6}\right]$ :

$$
\begin{aligned}
& \nabla \boldsymbol{e}=4 \pi G_{\varrho}, \\
& \nabla \times \boldsymbol{e}=-\frac{\partial}{\partial t} \boldsymbol{h}, \\
& \nabla \times \boldsymbol{h}=4 \pi G \boldsymbol{j}, \\
& \nabla \boldsymbol{h}=0 .
\end{aligned}
$$

Здесь @ и $\boldsymbol{j}$ - пока неизвестные величины, а операции дивергенции и роторы берутся в $\tilde{\boldsymbol{V}}_{3}$. Далее определим напряженности поля $\boldsymbol{e}$ и $\boldsymbol{h}$ следующим образом:

$$
\begin{aligned}
& \boldsymbol{e}=-\frac{\partial}{\partial t} \tilde{\boldsymbol{v}}+(1 / 2) \nabla(\tilde{\boldsymbol{v}} \tilde{\boldsymbol{v}}), \\
& \boldsymbol{h}=\nabla \times \tilde{\boldsymbol{v}} .
\end{aligned}
$$

В силу этих определений, как легко убедиться, соотношения (8) и (10) тождественно удовлетворяются, а из уравнений (7) и (9) получается соответственно

$$
\begin{aligned}
& \frac{\partial}{\partial t}(\nabla \tilde{\boldsymbol{v}})-(1 / 2) \nabla \nabla(\tilde{\boldsymbol{v}} \tilde{\boldsymbol{v}})+(1 / 2)(\nabla \times \tilde{\boldsymbol{v}})(\nabla \times \tilde{\boldsymbol{v}})=4 \pi G_{\varrho}, \\
& \nabla \times(\nabla \times \tilde{\boldsymbol{v}})=4 \pi G \boldsymbol{j},
\end{aligned}
$$

где

$$
\tilde{\varrho}=-\varrho+\frac{1}{8 \pi G}(\nabla \times \tilde{\boldsymbol{v}})(\nabla \times \tilde{\boldsymbol{v}}) .
$$


Видим, что если положить $\boldsymbol{j}=0$, то соотношения (13) и (14) эквивалентны уравнениям (3) и (4) соответственно для составляющих поля скоростей $\tilde{\boldsymbol{v}}$. Итак, при $\boldsymbol{j}=0$ уравнения (7)-(10) типа МаксвеллаЛоренца действительно эквивалентны уравнениям НР Г-поля (3)-(4). С учетом (11) и (12) уравнения движения (5) принимают вид, вполне аналогичный выражению лоренцевой силы в электродинамике, если последнее записать в надлежащей системе единиц для частицы с единичными массой и зарядом $\left[{ }^{5,6}\right]$ :

$$
\frac{\mathrm{d}}{\mathrm{d} t} \tilde{\mathbf{u}}=\boldsymbol{e}+[\tilde{\mathbf{u}} \times \boldsymbol{h}] .
$$

Если же условие (6) не имеет места, то можно дополнительно принять $\boldsymbol{h}=0$. Теперь уравнения (7), (8) и (16) описывают, очевидно, ньютоново НР Г-поле.

Отметим, что из общих уравнений НР Г-поля (2) можно было бы получить и случай $\mathbf{j} \neq 0$, но для этого мы должны располагать тензором энергии-импульса материи «экзотического» типа, таким, для которого в некоторых областях Г-поля в пределе $c \rightarrow \infty$ имело бы место $\tilde{\lambda}_{0 i} \neq 0$ и $\tilde{\lambda}_{i k}=0$.

3. Отделим друг от друга градиентную (потенциальную) и роторную (соленоидальную) части в векторном поле $\tilde{\boldsymbol{v}}$ :

$$
\tilde{\boldsymbol{v}}=\nabla \varphi+\boldsymbol{A}=\nabla \varphi+\nabla \times \boldsymbol{a} .
$$

(Величины $\varphi$ и $\boldsymbol{a}$ введены Х. Кересом $\left[{ }^{2}\right]$ как потенциалы поля скоростей $\tilde{\boldsymbol{v}})$. В силу определений (11) и (12) видим, что $\boldsymbol{A}$ является аналогом векторного, а величина

$$
U=\frac{\partial}{\partial t} \varphi-(1 / 2) \tilde{\boldsymbol{v}} \tilde{\boldsymbol{v}}
$$

- аналогом скалярного потенциала ЭМ-поля. Вместо $\boldsymbol{A}$ и $\boldsymbol{U}$ Х. Керес определяет потенциалы, так сказать, «классического гравитационного типа» - $\psi$ и $\Phi\left[{ }^{2}\right]$. В случае $\boldsymbol{j}=0$ связь вышеупомянутых потенциалов между собой и с напряженностями поля можно задать соотношениями $\left[{ }^{4}\right]$

$$
\begin{aligned}
& \boldsymbol{e}=-\nabla U-\frac{\partial}{\partial t} \boldsymbol{A}=-\nabla\left(\Phi-\psi^{2}\right)-\frac{\partial}{\partial t} \boldsymbol{A}, \\
& \boldsymbol{h}=\nabla \times \boldsymbol{A}=2 \nabla \psi .
\end{aligned}
$$

Уравнение (13) принимает вид

$$
\triangle \Phi=4 \pi G \tilde{\varrho},
$$

где $\Delta \equiv \nabla \nabla$ - оператор Лапласа, а уравнение (14) можно заменить условием интегрируемости соотношения $\nabla \times \boldsymbol{A}=2 \nabla \psi$ :

$$
\triangle \psi=0 .
$$

Условие (6) можно записать теперь в виде

$$
(\nabla \otimes \nabla) \psi \neq 0 .
$$

Отметим, что $\psi-$ аналог скалярного потенциала квазистационарного магнитного поля для «магнитовакуума». Отсюда, кстати, следует, что 
формулы и вычислительные методы, известные для этого частного вида магнитного поля (см., напр., $\left.\left[{ }^{7}\right]\right)$, формально переносятся на неньютонов случай НР Г-поля.

Поскольку для ньютонова Г-поля $\boldsymbol{h}=0$ и, следовательно, можно принять $\psi=0$, то в этом случае $\Phi$ и $U$ совпадают и обе величины могут толковаться как ньютоновы потенциалы. В общем неньютоновом случае понятие ньютонова потенциала по существу определяется неоднозначно: в качестве его можно толковать как $Ф$, так и $U$. Связь между «кулоновой» (ньютоновой) и «магнитной» (неньютоновой) компонентами НР Г-поля является на самом деле нелинейной. Например, вместо уравнения (21) получается более сложное уравнение для $U$ и $\psi$, которое по-прежнему переходит в известное уравнение Пуассона для чисто ньютонова поля, но теперь уже как $U$, так и $\psi$ связываются с плотностью̃ @ как источником НР Г-поля.

4. Исходя из аналогии с электродинамикой, приведенную выше теорию НР Г-полей можно развить также в лагранжевом и гамильтоновом формализмах. Например, применяя соответствующие формулы электродинамики $\left[{ }^{6}\right]$, получаем для частицы с единичной массой в НР Г-поле функции Лагранжа $L$ и Гамильтона $H$ в виде

$$
L=(1 / 2) \tilde{\boldsymbol{u}}^{2}-U+\boldsymbol{A} \tilde{\boldsymbol{u}}=(1 / 2) \tilde{\boldsymbol{u}}^{2}-\Phi+\psi^{2}+\boldsymbol{A} \tilde{\boldsymbol{u}}
$$

и

$$
H=(1 / 2) \tilde{\boldsymbol{u}}^{2}+U=(1 / 2) \tilde{\boldsymbol{u}}^{2}+\Phi-\psi^{2} .
$$

Из соотношения (25) видим, что в неньютоновом (вихревом) НР Г-поле отрицательная потенциальная энергия частицы по абсолютной величине больше, чем в ньютоновом поле с потенциалом Ф, следовательно, и сила притяжения к «вихревому гравитирующему центру» больше, чем сила тяготения к чисто ньютонову центру, определяющему потенциал Ф.

Для обобщенных импульса $\boldsymbol{P}$ и силы $\boldsymbol{F}$ имеем

$$
\begin{aligned}
& \boldsymbol{P}=\tilde{\boldsymbol{u}}+\boldsymbol{A}, \\
& \boldsymbol{F}=-\nabla U+(\tilde{\boldsymbol{u}} \nabla) \boldsymbol{A}+\tilde{\boldsymbol{u}} \times(\nabla \times \boldsymbol{A}) .
\end{aligned}
$$

Как известно, уравнение Гамильтона-Якоби получается заменой в функции Гамильтона $\boldsymbol{P}$ на $\nabla S$, а $H-$ на $-\frac{\partial}{\partial t} S:$

$$
-\frac{\partial}{\partial t} S=\frac{1}{2}[\nabla S-\boldsymbol{A}]^{2}+\Phi-\psi^{2}
$$

В силу соотношений (17) и (18) это уравнение тождественно удовлетворяется, если принять

$$
S=-\varphi
$$

Таким образом, в качестве частного решения уравнения (28) скалярный потенциал $\varphi$ поля скоростей $\tilde{\boldsymbol{v}}$ можно интерпретировать как функцию действия (с обратным знаком) для свободной пробной частицы в соответствующем НР Г-поле.

В заключение автор выражает благодарность П. Карду за внимание к работе и обсуждение результатов. 
ЛИТЕРАТ У РА

1. Кере с Х., ЖЭТФ, 46, 1741 (1964).

2. К е р е с Х., ЖЭТФ, 48, 1319 (1965).

3. Кер ес Х., Изв. АН ЭССР, Физ. Матем., 25, 349 (1976).

4. К оппель А., Нерелятивистские гравитационные поля в общей теории относительности, Ротапринт ТГУ, 1977.

5. Л анд ау Л. Д., Лифшиц Е. М., Теория поля, М., 1973, с. 70, 91.

6. Л ев ич В. Г., Курс теоретической физики I, М., 1969, с. 41, 43, 182.

7. Gordo n, W. B., J. Math. Phys., 16, 448 (1975).

Тартуский государственный университет

Поступила в редакцию 16/XII 1976

\title{
ДЕПОНИРОВАННЫЕ СТАТЬИ
}

\author{
EESTI NSV TEADUSTE AKADEEMIA TOIMETISED, 26. KOIDE \\ FOOSIKA * MATEMAATIKA. 1977, NR. 3
}

ИЗВЕСТИЯ АКАДЕМИИ НАУК ЭСТОНСКОИ ССР. ТОМ 26 ФИЗИКА * МАТЕМАТИКА, 1977, № 3

\section{КОНЪЮНКЦИЯ и ДИЗЪЮНКЦИЯ СИГНАЛОВ В имПУЛЬСНОЙ СТРУКТУРЕ}

Опираясь на аппарат векторно-переменных переключательных функций (ВП-функций) как на язык для аналитического описания логических схем, рассмотрены все возможные виды схемной реализации конъюнкции и дизъюнкции сигналов минимальными логическими схемами в импульсной элементной структуре.

Определены два вида конъюнкции сигналов: обычная конъюнкция (т. е. совпадение сигналов) и конъюнкция, инвариантная ко времени существования сигналов - ее аргументов (т. е. конъюнкция разновременных сигналов). Сигналы-аргументы либо существуют сами, либо существуют их отрицания.

Указанные выше задачи реализуются на комбинационных или накапливающих схемах. В последнем случае используются логические схемы на отдельных триггерах и на целых сдвиговых регистрах.

Рукопись депонирована Институтом кибернетики АН ЭССР в ВИНИТИ, № 4490-76 от 21/XII 1976, с. 26, библ. 19 назв.

Реферат поступил в редакцию 4/II 1977. 